\title{
THE SIMPLE GROUPS RELATED TO $M_{24}$, II
}

Dedicated to the memory of Hanna Neumann

\author{
DIETER HELD
}

(Received 13 March 1972)

Communicated by M. F. Newman

The objective of this paper is to prove the following generalization of the main result in [2]:

THEOREM. Let $G$ be a finite simple group which possesses an involution $t$ such that the centralizer of $t$ in $G$ is isomorphic to the centralizer of an involution in $H$. Then $G$ is isomorphic to $L_{5}(2), M_{24}$, or $H$.

REMARK. By $H$ we denote a simple group of order $2^{10} \cdot 3^{3} \cdot 5^{2} \cdot 7^{3} \cdot 17$ discussed in [2]. The group $H$ has precisely two classes of involutions. If $C(t)$ is isomorphic to the centralizer of a 2-central involution of $H$, the result follows from [2; Theorem. p. 253]. Thus, in what follows, we shall assume that $C(t)$ is isomorphic to the centralizer of an involution of $H$ which is not contained in the center of a $S_{2}$-subgroup of $H$. Using the characterization of $L_{5}(2), M_{24}$, and $H$ by their Sylow 2-subgroups due to. Schoenwaelder [3], Deckers [1] has shown that our theorem holds in case that a $S_{2}$-subgroup of $G$ has order at most $2^{10}$. Thus, we shall assume that $2^{11}$ divides the order of $G$.

Familiarity with section 1 and with the lemmas 3.2-3.6 of [2] will be assumed. Table II of [2; p. 279] lists the conjugacy classes of $\boldsymbol{C}(t)$. We use the notation introduced in [2]. In particular, a $S_{2}$-subgroup $S$ of $C(t)$ will be described by generators and relations given in section 1 of [2]. We put

$$
S=\left\langle z_{1}, z_{3}, z_{4}, \pi, \tau, \mu, \lambda, \mu^{\prime} \tau^{\prime}, z_{2} \tau^{\prime}\right\rangle
$$

and $t=z_{3} \pi$. The four-group $V=\left\langle z_{3} \pi, z_{1} \mu \tau\right\rangle$ is normal in $C(t)$ and $C(t) / V$ contains a unique subgroup $H^{*} / V$ of index 2 isomorphic to $L_{3}(4)$. As in [2; Lemma 2.2, p. 257] put $R_{1}=\left\langle z_{1}, z_{3}, z_{4}, \pi, \mu \tau, \mu^{\prime} \tau^{\prime}\right\rangle$ and $R_{2}=\left\langle z_{1}, z_{3}, \pi, \mu, \tau, \lambda\right\rangle$. Then $R_{1} R_{2}$ is a $S_{2}$-subgroup of $H^{*}$. Since $z_{2} \tau^{\prime}$ does not centralize $V$, we have $C(V)=H^{*}$. Note that $C(V)$ does not split over $V$.

It seems worthwhile remarking that $C_{H}\left(z_{3} \pi\right)$ is very similar to the centralizer of an involution in the Suzuki sporadic group. 


\section{Preliminary lemmas}

LEMMA 1.1. Under the action of $N\left(R_{i}\right) \cap C\left(z_{3} \pi\right), i=1,2$, we have the following classes of involutions in $R_{i}$ : There is one conjugate of $z_{3} \pi$, there are two conjugates of $z_{1} \mu \tau$, there are 15 conjugates of $z_{1}$, there are 15 conjugates of $z_{1} z_{3} \pi$, and there are 30 conjugates of $\pi$.

PRoof. The assertion follows immediately from [2; lemma 3.2, p. 271 , and table II, p. 279].

LEMMA 1.2. For $i=1,2$, the group $N\left(R_{i}\right) \cap C\left(z_{3} \pi\right)$ is a splitting extension of $R_{i}$ by $\Sigma_{5}$. We have $Z(S)=\left\langle z_{1}, z_{3} \pi\right\rangle$ and $\left\langle z_{1}\right\rangle$ char $S$. The involutions $z_{3} \pi$ and $z_{1} z_{3} \pi$ are conjugate in every subgroup of order $2^{10}$ of $G$ which contains $S$. There are only the following possibilities for the fusion of involutions of $R_{i}$ under the action of $N\left(R_{i}\right), i=1$ or 2:

$$
C_{z_{3} \pi}
$$

(a) $z_{3} \pi \sim z_{1} z_{3} \pi$

(b) $z_{3} \pi \sim z_{1} z_{3} \pi \sim z_{1} \mu \tau$

(c) $z_{3} \pi \sim z_{1} z_{3} \pi \sim \pi$

(d) $z_{3} \pi \sim z_{1} z_{3} \pi \sim z_{1} \mu \tau \sim \pi$

(e) $z_{3} \pi \sim z_{1} \mu \tau$

(f) $z_{3} \pi \sim \pi$

(g) $z_{3} \pi \sim z_{1} \mu \tau \sim \pi$

(b) $z_{3} \pi$
16

18

46

48

3

31

33

1
$\left|N\left(R_{i}\right)\right|$

$2^{13} \cdot 3 \cdot 5$

$2^{10} \cdot 3^{3} \cdot 5$

$2^{10} \cdot 3 \cdot 5 \cdot 23$

$2^{13} \cdot 3^{2} \cdot 5$

$2^{9} \cdot 3^{2} \cdot 5$

$2^{9} \cdot 3 \cdot 5 \cdot 31$

$2^{9} \cdot 3^{2} \cdot 5 \cdot 11$

$2^{9} \cdot 3 \cdot 5$

Proof. The assertions follow easily from lemma 1.1, the fact that $S^{\prime \prime}=\left\langle z_{1}\right\rangle$, and [2; lemma 3.2].

LemMa 1.3. The cases (c), (f), and (g) are not possible.

Proof. Since 11 and 23 do not divide the order of $L_{6}(2)$, we see that (c) and (g) cannot happen. If we are in case (f), then an element of order 31 acts trivially on the $N\left(R_{i}\right)$-class of $z_{1}$ because $z_{1} \sim z_{3} \pi$ is impossible by (1.2).

LEMMA 1.4. If a $S_{2}$-subgroup of $N\left(R_{i}\right)$ for $i=1$ or $i=2$ has order $2^{9}$ we get a contradiction.

Proof. Assume that $\left|N\left(R_{i}\right)\right|=2^{9} \cdot 3 \cdot 5$ or $2^{9} \cdot 3^{2} \cdot 5$ for one $i \in\{1,2\}$. Denote by $S^{*}$ a 2 -subgroup of $G$ which contains $S$ as a subgroup of index 2 . There is an element $f \in S^{*}$ such that $S^{*}=S\langle f\rangle$ and $R_{1}{ }^{f}=R_{2}$. Also we have $\left(z_{3} \pi\right)^{f}=z_{1} z_{3} \pi$. If $\left|N\left(R_{i}\right)\right|=2^{9} \cdot 3 \cdot 5$, then $\left[f, z_{3} \pi\right]=1$ which is impossible. Let $\left|N\left(R_{i}\right)\right|=2^{9} \cdot 3^{2} \cdot 5$ We may assume $i=1$. Then $f$ maps the $N\left(R_{1}\right)$-class of $z_{3} \pi$ onto the $N\left(R_{2}\right)$-class of $z_{1} z_{3} \pi$. However, in $N\left(R_{1}\right)$ the element $z_{3} \pi$ has precisely three conjugates 
whereas in $N\left(R_{2}\right)$ the element $z_{1} z_{3} \pi$ has at least 15 conjugates. We have arrived at a contradiction.

LEMMA 1.5. $A S_{2}$-subgroup of $N\left(R_{i}\right), i=1,2$, has order at least $2^{10}$. We are in case (a), (b), or (d).

Proof. This follows from the above lemmas.

LEMMA 1.6. Let $T$ be a subgroup of $G$ of order $2^{10}$ which contains $S$. Then, $R_{1}$ and $R_{2}$ are the only elementary abelian subgroups of $T$ which have order 64.

Proof. We have $Z(T)=\left\langle z_{1}\right\rangle$. Denote by $R_{3}$ an elementary abelian subgroup of $T$ with $R_{1} \neq R_{3} \neq R_{2}$ and $\left|R_{3}\right|=64$. Thus $S R_{3}=T$ and $\left|S \cap R_{3}\right|$ $=2^{5}$. As $S \cap R_{3} \supseteq R_{1} R_{2} \cap R_{3}$ and $2^{10} \geqq\left|R_{1} R_{2} R_{3}\right| \geqq 2^{9}$, we must have $2^{5} \geqq\left|R_{1} R_{2} \cap R_{3}\right| \geqq 24$. An involution of $R_{1} R_{2}$ lies in $R_{1}$ or in $R_{2}$. Let $r_{1}, r_{2}$ be involutions contained in $R_{1} R_{2} \cap R_{3}$ such that $r_{1} \in R_{1}$ and $r_{2} \in R_{2} \mid R_{1}$. Clearly, $r_{1} \in C_{R_{1}}\left(r_{2}\right)=R_{1} \cap R_{2} \subset R_{2}$. Thus, $\left\langle r_{1}, r_{2}\right\rangle \subseteq R_{2}$. It follows $R_{1} R_{2} \cap R_{3} \subseteq R_{i}$ $i=1$ or 2 , and so, $R_{1} R_{2} \cap R_{3}=R_{3} \cap R_{i}, i=1$ or 2 . Assume first that $\left|R_{1} R_{2} \cap R_{3}\right|=2^{4}$. Then, $\left|R_{1} R_{2} R_{3}\right|=2^{10}$. Without loss of generality we may assume that $R_{1} R_{2} \cap R_{3}=R_{3} \cap R_{1}$. Clearly, $\left|R_{2} \cap R_{3}\right| \geqq 2^{2}$ and $R_{2}$ $\cap R_{3} \subseteq R_{1} R_{2} \cap R_{3}=R_{3} \cap R_{1}$. Thus, $R_{2} \cap R_{3}$ would be in $Z(T)$ which is impossible. Assume finally that $\left|R_{1} R_{2} \cap R_{3}\right|=2^{5}$. Then, $\left|R_{1} R_{2} R_{3}\right|=2^{9}$. Without loss of generality we may assume that $R_{1} R_{2} \cap R_{3}=R_{1} \cap R_{3}$. Clearly, $\left|R_{2} \cap R_{3}\right| \geqq 2^{3}$ and $R_{2} \cap R_{3} \subseteq R_{1} R_{2} \cap R_{3}=R_{1} \cap R_{3}$. Thus, $R_{2} \cap R_{3}$ lies in $Z\left(R_{1} R_{2} R_{3}\right)$. But $\left|T: R_{1} R_{2} R_{3}\right|=2$. From the Jordan-canonical form for an element of $T \backslash R_{1} R_{2} R_{3}$ on $\Omega_{1}\left(Z\left(R_{1} R_{2} R_{3}\right)\right)$, we get a contradiction to $|Z(T)|=2$. The lemma is proved.

LEMMA 1.7. If $\omega$ is an element of order 5 of $C\left(z_{3} \pi\right)$, then a $S_{2}$-subgroup of $C(\omega)$ has order $2^{2}$ and a $S_{2}$-subgroup of $N(\langle\omega\rangle)$ has order $2^{4}$.

Proof. We know that $\boldsymbol{C}\left(z_{3} \pi\right)$ contains a subgroup isomorphic to $\Sigma_{5}$. Clearly, $V$ lies in $\boldsymbol{C}(\omega)$. Let $V^{*}$ be a subgroup of order 8 of $\boldsymbol{C}(\omega)$ such that $V \subset V^{*}$. If $z_{3} \pi \approx z_{1} \mu \tau$ in $G$, we get $z_{3} \pi \in Z\left(V^{*}\right)$. If $z_{3} \pi \sim z_{1} \mu \tau$, then $Z\left(V^{*}\right)$ contains an involution conjugate to $z_{3} \pi$ in $G$. It follows that $V$ must be a $S_{2}$-subgroup of $C(\omega)$.

\section{The case (b)}

In this section we suppose that a $S_{2}$-subgroup of $N\left(R_{i}\right)$ has order $2^{10}$ for one $i \in\{1,2\}$.

LeMMA 2.1. For $i=1$ and $i=2$ we have $\left|N\left(R_{i}\right)\right|=2^{10} \cdot 3^{3} \cdot 5$.

Proof. Without loss of generality let $\left|N\left(R_{1}\right)\right|=2^{10} \cdot 3^{3} \cdot 5$. Denote by $T$ a $S_{2}$-subgroup of $N\left(R_{1}\right)$ with $S \subset T$. From (1.6) we get that $R_{1}$ and $R_{2}$ are the 
only elementary abelian subgroups of order 64 of $T$. Denote by $T^{*}$ a subgroup of order $2^{11}$ of $G$ with $T \subset T^{*}$. There is an element $f \in T^{*} \mid T$ such that $R_{1}{ }^{f}=R_{2}$. The lemma is proved.

Lemma 2.2. The order of $N\left(R_{1} R_{2}\right)$ is $2^{11} \cdot 3^{2}$.

Proof. From (1.6) we get that $2^{11}$ divides the order of $N\left(R_{1} R_{2}\right)$. Since all involutions of $V$ are conjugate, we get $|N(V)|=2^{9} \cdot 3^{3} \cdot 5 \cdot 7$. We know that $R_{1} R_{2}$ is a $S_{2}$-subgroup of $C(V)$ and that $\left|N\left(R_{1} R_{2}\right) \cap C(V)\right|=2^{8} \cdot 3$. The Frattiniargument yields $N(V)=\left(N\left(R_{1} R_{2}\right) \cap N(V)\right) C(V)$. It follows that $N\left(R_{1} R_{2}\right)$ contains an elementary abelian group of order 9 . Also one has $\left|N\left(R_{1} R_{2}\right) \cap C\left(z_{3} \pi\right)\right|$ $=2^{9} \cdot 3$. It follows $\left|N\left(R_{1} R_{2}\right)\right|=2^{11} \cdot 3^{2}$, since $z_{3} \pi$ cannot have more than 12 conjugates under the action of $N\left(R_{1} R_{2}\right)$.

LEMMA 2.3. The involution $z_{1}$ has precisely three conjugates under the action of $N\left(R_{1} R_{2}\right)$.

Proof. From (1.2) we see that $z_{1}$ lies in the center of a $S_{2}$-subgroup of $N\left(R_{1} R_{2}\right)$. Assume by way of contradiction that $3^{2}$ divides $\left|C\left(z_{1}\right) \cap N\left(R_{1} R_{2}\right)\right|$. We know that $N\left(R_{1} R_{2}\right)=N\left(R_{1} \cap R_{2}\right)$ and that $C\left(R_{1} \cap R_{2}\right)=R_{1} R_{2}$. Our assumption implies that an element of order 3 centralizes $R_{1} \cap R_{2}$ which is not possible. Since $z_{3} \pi$ has 12 conjugates in $R_{1} \cap R_{2}$ under $N\left(R_{1} R_{2}\right)$, we see that $z_{1}$ has precisely three conjugates under the action of $N\left(R_{1} R_{2}\right)$.

Lemma 2.4. In $N\left(R_{1} R_{2}\right)$ we have $z_{1} \sim \pi$.

ProOF. Assume by way of contradiction that there is an element $g$ in $N\left(R_{1} R_{2}\right)$ such that $\left(z_{3} \pi\right)^{g}=\pi$. Then, $R_{1}^{g}=R_{2}$. This is impossible, since in $N\left(R_{1}\right)$ the element $z_{3} \pi$ has only 18 conjugates, whereas in $N\left(R_{2}\right)$ the element $\pi$ has at least 30 conjugates. Hence, $z_{3} \pi \sim \pi$ holds in $N\left(R_{1} R_{2}\right)$. Thus, $z_{1} \sim \pi$ in $N\left(R_{1} R_{2}\right)$.

Lemma 2.5. The case (b) is not possible.

Proof. The only elements of $R_{1} \cap R_{2}$ conjugate to $z_{1}$ are $z_{1}, \pi, z_{1} \pi$. All other involutions of $R_{1} \cap R_{2}$ are conjugate to $z_{3} \pi$ under $N\left(R_{1} R_{2}\right)$. We know that $V \tau$ is conjugate to $V z_{1}$ in $N(V)$. All elements of $V z_{1}$ except $z_{1}$ are conjugate to $z_{3} \pi$ in $G$. The coset $R_{1} \tau$ has precisely 16 involutions which fuse under $S$ in the following way:

$$
\begin{aligned}
& \tau \sim z_{1} \tau \sim \pi \tau \sim z_{1} \pi \tau, \\
& z_{3} \tau \sim z_{1} z_{3} \tau \sim z_{3} \pi \tau \sim z_{1} z_{3} \pi \tau, \\
& \mu \sim z_{1} \mu \sim z_{3} \pi \mu \sim z_{1} z_{3} \pi \mu, \\
& \pi \mu \sim z_{1} \pi \mu \sim z_{1} z_{3} \mu \sim z_{3} \mu .
\end{aligned}
$$

Hence, $R_{1} \tau$ contains at least eight involuticns conjugate to $z_{3} \pi$ in $G$. Thus $R_{2}$ contains at least 20 conjugates of $z_{3} \pi$. Since under the action of $N\left(R_{2}\right)$ the ele- 
ment $z_{3} \pi$ has only 18 conjugates, we get $z_{3} \pi \sim z_{1}$ as $z_{1} \sim \pi$ which is impossible. The lemma is proved.

\section{The cases (a) and (d)}

In this section we finish the proof of our theorem. We know that $2^{13} \cdot 3 \cdot 5$ divides $\left|N\left(R_{i}\right)\right|$ and that $\left|N\left(R_{i}\right)\right|$ divides $2^{13} \cdot 3^{2} \cdot 5$ for $i=1$ and $i=2$. Remember that $N\left(R_{i}\right)$ contains a subgroup $\Sigma$ isomorphic to $\Sigma_{5}$.

We study the structure of $N\left(R_{i}\right)$. Put $R=N\left(R_{i}\right) / R_{i}$. We may choose $\Sigma$ to be a subgroup of $C\left(z_{3} \pi\right)$. Then, $\left[\Sigma^{\prime}, V\right]=\langle 1\rangle$. The only simple groups which can be involved in $R$ are $A_{5}$ and $A_{6}$. It is a consequence of $(1.7)$ that $O_{2}(R) \neq\langle 1\rangle$. Denote by $L$ the inverse image of $O_{2}(R)$ in $N\left(R_{i}\right)$. Then, $|L| \geqq 2^{7}$. Because of $C\left(R_{i}\right)=R_{i}$, we get $Z(L) \subset R_{i}$. Obviously, $L \cap C\left(z_{3} \pi\right)=R_{i}$ because $\Sigma \cap L=\langle 1\rangle$. Assume that $Z(L)$ lies in $V$. Then, $|Z(L)|=2$, and $Z(L)$ is centralized by $\Sigma$. This would imply $Z(L)=\left\langle z_{3} \pi\right\rangle$, a contradiction. Hence, $Z(L) \nsubseteq V$. It follows that $\Sigma^{\prime}$ is faithfully represented on $Z(L)$. Thus, $|Z(L)| \geqq 2^{4}$. Assume that $V \cap Z(L) \neq\langle 1\rangle$. This intersection does not contain $\left\langle z_{3} \pi\right\rangle$. Hence, $Z(L) \cap V$ is equal to $\left\langle z_{1} \mu \tau\right\rangle$ or to $\left\langle z_{1} z_{3} \pi \mu \tau\right\rangle$. But $z_{2} \tau^{\prime}$ normalizes $Z(L) \cap V$. This gives a contradiction. We have shown that $R_{i}=V \times Z(L)$. Since $\Sigma^{\prime}$ normalizes $Z(L)$ we get that $V \times Z(L) \Sigma^{\prime}$ splits over $V$. This is not possible. The theorem is proved.

\section{References}

[1] M. Deckers, 'Simple groups having centralizers of involutions isomorphic to those in $\mathrm{He}^{\text {', }}$ Archiv Math. (To appear).

[2] D. Held. 'The simple groups related to $M_{24}$ ', J. Algebra 13 (1969), 253-296.

[3] U. Schoenwaelder, 'Finite groups with a Sylow 2-subgroup of type $\mathrm{M}_{24}$, J. Algebra (To appear).

\section{Department of Mathematics}

University of Mainz

6500 Mainz

Germany 